\title{
Stimulus variation and dimensional contrast
}

\author{
JOHN M. HINSON and JENNIFER J. HIGA \\ Washington State University, Pullman, Washington
}

\begin{abstract}
In two experiments, a maintained generalization procedure was employed to examine stimulus control of pigeons' responses to a visual wavelength continuum. For both experiments, pigeons' responses were periodically reinforced during wavelength values from one end of a continuum, while responses during other stimulus values were extinguished. In Experiment 1, the set of positive stimulus values remained constant, while the spacing of the set of negative stimuli varied. In Experiment 2, the set of negative stimulus values remained constant, while the spacing of positive stimuli varied. Positive dimensional contrast effects were obtained in both experiments. In general, the results indicated that variation in the spacing of negative stimuli had little effect on positive dimensional contrast. However, variation in the spacing of positive stimuli produced changes in the peak of the dimensional contrast gradient, without apparent change in the mag nitude of the effect.
\end{abstract}

One of the more prominent results from maintained generalization studies of dimensional stimulus control is dimensional contrast (see D. S. Blough, 1975; Catania \& Gill, 1964; Malone \& Staddon, 1973; Reynolds, 1961). Dimensional contrast refers to the relative enhancement of response differences in border regions of empirical stimulus control gradients. More specifically, positive dimensional contrast refers to increased responding during positive $(S+)$ stimuli near the border with negative $(\mathrm{S}-$ ) stimuli, relative to $\mathbf{S}+$ stimuli farther removed from $\mathrm{S}-$. Negative dimensional contrast refers to decreased responding during $\mathbf{S}$ - stimuli near the border with $\mathbf{S}+$, relative to $S$ - stimuli farther removed from $S+$.

Dimensional contrast effects have been observed in a variety of discrimination tasks, including ones involving spatial orientation stimuli (e.g., Catania \& Gill, 1964; Malone, 1975; Reynolds, 1961), visual wavelength stimuli (D. S. Blough, 1975), auditory amplitudes (P. M. Blough, 1980), and visual flicker rates (Hinson, 1988). Furthermore, the effects are dependent on relative rates of reinforcement during $\mathrm{S}+$ and $\mathrm{S}-$, rather than on the presence or absence of reinforcement (D. S. Blough, 1975). The similarity of dimensional contrast effects to peak shift effects has been noted by several authors (e.g., D. S. Blough, 1975; Honig \& Urcuioli, 1981). However, it never has been clearly demonstrated that these effects are produced by the same variables.

It is difficult to reconcile dimensional contrast effects with widely accepted assumptions concerning stimulus generalization and discrimination (see D. S. Blough,

This research was supported by funds provided by National Institute of Mental Health Grant MH40275-01 to Washington State University. Experiment 1 was part of a dissertation submitted by Jennifer J. Higa in partial fulfillment of the requirements for the doctoral degree at Washington State University. We thank John Malone for helpful comments on an earlier version of this paper. Address reprint requests to J. M. Hinson, Department of Psychology, Washington State University, Pullman, WA 99164-4830.
1983). In particular, dimensional contrast indicates a certain inconsistency in the relationship between stimulus similarity and stimulus discrimination performance; that is, $\mathbf{S}-$ and $\mathbf{S}+$ values that are more similar to one another appear to be discriminated better than less similar values. The absence of monotonicity between stimulus similarity and discrimination performance measures is contrary to the predictions of most popular theories of discrimination learning (D. S. Blough, 1983; Hinson, 1988).

Based on the studies available, the level of discrimination between $\mathrm{S}+$ and $\mathrm{S}$ - appears to be a crucial factor in producing dimensional contrast. The importance of this factor has been illustrated indirectly in several reports. For example, some studies have shown that dimensional contrast appears at an early stage of training and diminishes over time (e.g., P. M. Blough \& D. S. Blough, 1985; Farthing, 1974; Hinson \& Malone, 1980; Malone, 1975). The apparent absence of dimensional contrast af ter extended training could be due to a floor or ceiling effect in dependent measures when discrimination is complete (Hinson \& Malone, 1980). Moreover, dimensional contrast appears and disappears more rapidly with easier discriminations (Malone, 1975; Malone \& Rowe, 1981; Rowe \& Malone, 1981). Finally, the effect can be reinstated, or its magnitude increased, by altering stimulus values to make them less discriminable (e.g., Catania \& Gill, 1964; Malone, 1975).

Results of more direct studies indicate that changes in the spacing between positive and negative stimuli, which should increase discriminability, can have a marked effect on dimensional contrast. For example, elimination of a sufficient number of positive and negative stimuli along the border of a stimulus continuum can diminish dimensional contrast (P. M. Blough, 1980). However, elimination of border stimuli in a difficult discrimination task can enhance dimensional contrast (Hinson, 1988). Thus, increased discrimination between $\mathbf{S}+$ and $\mathbf{S}-$ may either increase or decrease dimensional contrast, depend- 
ing on the previous level of discrimination. If the level of discrimination between $\mathrm{S}-$ and $\mathrm{S}+$ is either too low or too high, dimensional contrast will not be observed.

Concern with variables governing discrimination between $S+$ and $S-$ is consistent with the theoretical emphasis given these variables. Such prominent theories of discrimination as the interacting gradient model (e.g., Spence, 1937), the signal detection model (e.g., Boneau \& Cole, 1967; Heinemann \& Chase, 1975), and the incremental model (e.g., D. S. Blough, 1975) all base predictions largely on the degree of separation between $\mathbf{S}+$ and $\mathbf{S}-$ along the stimulus continuum. Less attention has been paid to other factors, such as those affecting discrimination within each set of $\mathrm{S}-$ and $\mathrm{S}+$ stimulus values.

The present experiments were designed to assess the effects of variation within either $S-$ or $S+$ stimulus sets on positive dimensional contrast. All studies involved discrimination by pigeons of visual wavelength values presented during a maintained generalization procedure. In the first experiment, $\mathbf{S}+$ stimulus values were held constant while $S-$ stimuli varied during different conditions. A second experiment held $S-$ stimuli constant while $S+$ values varied across conditions.

\section{EXPERIMENT 1}

\section{Method}

Subjects. Four male homing pigeons served as subjects. One of the subjects, Bird 3, was experimentally naive, whereas the others had varied histories that did not include wavelength discrimination training. The subjects were maintained at $80 \%$ of their laboratory, free-feeding weights.

Apparatus. Experiments were carried out in a standard Plexiglas and aluminum operant conditioning chamber. The internal dimensions of the chamber were $36 \times 37 \times 35 \mathrm{~cm}$. A Campden Instruments translucent pecking key, $2.5 \mathrm{~cm}$ in diameter, was affixed to the center of the front wall about $21.5 \mathrm{~cm}$ above the floor. A 6-W houselight located in the upper left comer of the front panel provided diffuse illumination throughout the chamber. A $6 \times 7 \mathrm{~cm}$ aperture, $12 \mathrm{~cm}$ below the key, provided access to a food magazine. Access to mixed grain for $3 \mathrm{sec}$ was used as a reinforcer. The experimental chamber was housed in a larger soundproofing box that contained a fan for ventilation and masking of extraneous noise. Experimental events and data collection were controlled by a microcomputer in the same room.

Wavelength stimuli were produced by a Photon Technology, .25 -m diffraction-grating monochromator. Wavelength selection was by means of a computer-controlled, precision stepper motor. An Oriel 100-W quartz halogen source, operated at $12 \mathrm{~A}$, passed light through the monochromator entrance and exit slits, which were set to provide a half bandwidth of $5 \mathrm{~nm}$. The output image was focused by means of a lens onto the back of the response key, and appeared as a $3 \times 2.5 \mathrm{~cm}$ rectangle. The luminance of all stimuli was approximately $32 \mathrm{~cd} / \mathrm{m}^{2}$.

Procedure. After preliminary autoshaping in the chamber for both naive and experienced birds, the subjects were exposed to a maintained generalization procedure. The stimuli ranged from 572 to $618 \mathrm{~nm}$. Wavelengths lower than $596 \mathrm{~nm}$ were designated $S-$, and responses during these were never reinforced. Stimuli higher than or equal to $596 \mathrm{~nm}$ were designated $\mathrm{S}+$, and responses during these were reinforced according to a variable-interval 60 -sec schedule. A single session comprised 12030 -sec stimulus presentations. The sequence of stimuli was determined by a pseudorandom process and changed daily.

For baseline, only $S+$ values appeared throughout the session, whereas for three discrimination conditions, both $S+$ and $S-$ values were presented. The stimulus values, in nanometers, for baseline and discrimination conditions appear in Table 1. During discrimination conditions, the probability of presentation of $S+$ and $S-$ was the same, and the total number of distinct stimulus values was constant at 12 for $S+$ and 4 for $S-$. However, although the spacing of $S+$ values remained the same in all conditions, the spacing of S- varied. In Condition 1, all S- values were spaced closely along the continuum with $\mathrm{S}+$, whereas in Condition 3 , all $\mathrm{S}-$ values were spaced away from $S+$. Condition 2 included $S-$ stimuli that were both close to and far from $S+$. The order of conditions and the number of daily sessions of training for each subject appear in Table 2.

\section{Results}

Figure 1 provides maintained generalization gradients for individual subjects in each discrimination condition. Each discrimination condition is represented by a function with a unique symbol: an upright cross for Condition 1 , a circle for Condition 2 , and an asterisk for Condition 3. To minimize the influence of differences in absolute response rate, the gradients are scaled in terms of the maximum response rate occurring in each condition. The horizontal axis of the graphs is scaled in 2-nm spacings. Because the rate of discrimination learning differed among individuals, the gradients represent the first seven consecutive sessions of stable performance during each condition. Stability was defined in terms of two sets of criteria. First, changes in daily absolute response rate had to be less than $10 \%$, and there could be no obvious trend of increase or decrease. Second, the daily peak of the gradient could vary by no more than \pm 1 stimulus position (i.e., $2 \mathrm{~nm}$ ) from the peak of the mean gradient.

The individual maintained gradients in Figure 1 show positive dimensional contrast for every case, except Condition 1 for Bird 4. Peaks in the gradients are at intermediate values of $S+$, usually between 606 and $610 \mathrm{~nm}$. The magnitude of positive dimensional contrast is indi-

Table 1

Wavelength Values (in $\mathrm{nm}$ ) for $\mathrm{S}+$ and $\mathrm{S}-$, in Each Condition of Experiment 1

\begin{tabular}{cccccc}
\hline & & & \multicolumn{3}{c}{$S-$ Condition } \\
\cline { 4 - 6 } & S+ & & 1 & 2 & 3 \\
\hline 596 & 604 & 612 & 588 & 572 & 572 \\
598 & 606 & 614 & 590 & 574 & 574 \\
600 & 608 & 616 & 592 & 592 & 576 \\
602 & 610 & 618 & 594 & 594 & 578 \\
\hline
\end{tabular}

Table 2

Order of Conditions and Number of Sessions for Each Condition of Experiment 1

\begin{tabular}{cc}
\hline Bird & Condition (Sessions) \\
\hline 1 & Baseline (1-18), C2 (19-46), C1 (47-74), C3 (75-103) \\
2 & Baseline (1-18), C2 (19-46), C1 (47-74), C3 (75-103) \\
3 & Baseline (1-18), C1 (19-46), C2 (47-74), C3 (75-103) \\
4 & Baseline (1-18), C1 (19-46), C2 (47-74), C3 (75-103) \\
\hline
\end{tabular}




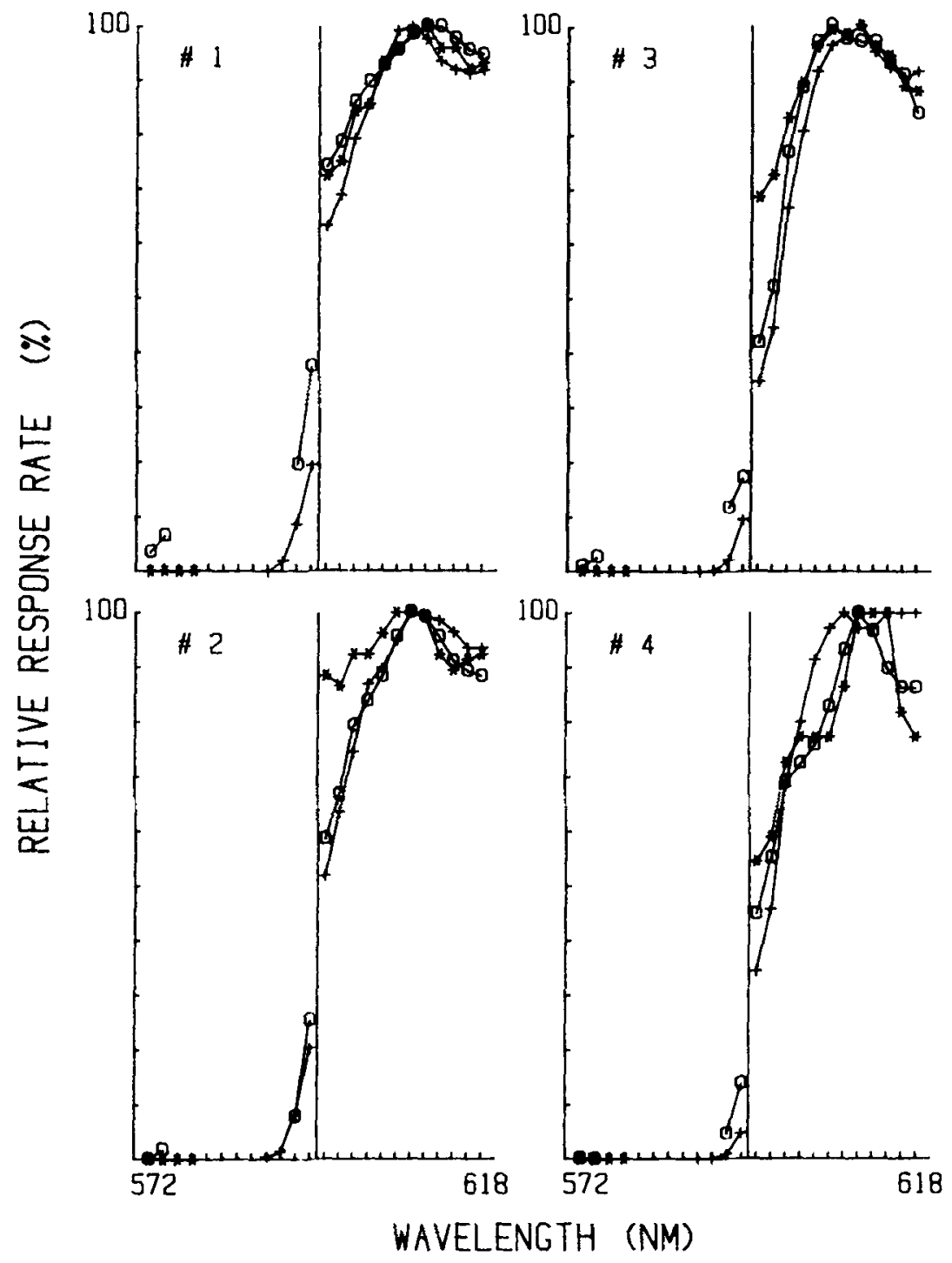

Figure 1. Maintained generalization gradients for the 4 birds in Experiment 1. The vertical axis of the gradients represents the mean relative response rate for the first seven consecutive sessions of stable performance during each discrimination condition. Symbols for each condition are an upright cross for Condition 1, an open circle for Condition 2, and an asterisk for Condition 3. The horizontal axis is scaled in 2-nm divisions. The perpendicular line between values 594 and $596 \mathrm{~nm}$ shows the division between Sand $\mathbf{S}+$.

cated by the difference in response rate between the $S+$ value that produces peak responding (approximately $608 \mathrm{~nm})$ and the most extreme value of $S+(618 \mathrm{~nm})$. The range of differences among subjects is from about $10 \%$ to $25 \%$ of the maximum response rate.

Figure 2 provides a set of mean maintained gradients for the group of subjects in Experiment 1. This figure clearly shows that there is no obvious effect of the discrimination condition on the form or magnitude of positive dimensional contrast. Despite changes in the spacing of the set of S- stimuli, peak responding in S+ occurs at about the same stimulus value. Moreover, the differ- ence in response rate between peak and extreme $S+$ stimulus values is roughly equal across conditions.

However, this does not mean that changing the spacing of the S- stimulus set had no effect on discrimination. In fact, there is a consistent effect of moving Sstimuli away from the border with $S+$. In all cases, relative response rate is higher during border $\mathrm{S}+$ stimuli (i.e., $596-600 \mathrm{~nm}$ ) in Condition 3 than in Condition 1, with Condition 2 response rates falling somewhere in between. This ordinal relation also appears in the individual gradients of Figure 1, with the exception of Bird 1, for whom Conditions 2 and 3 had roughly the same effect. Thus, 


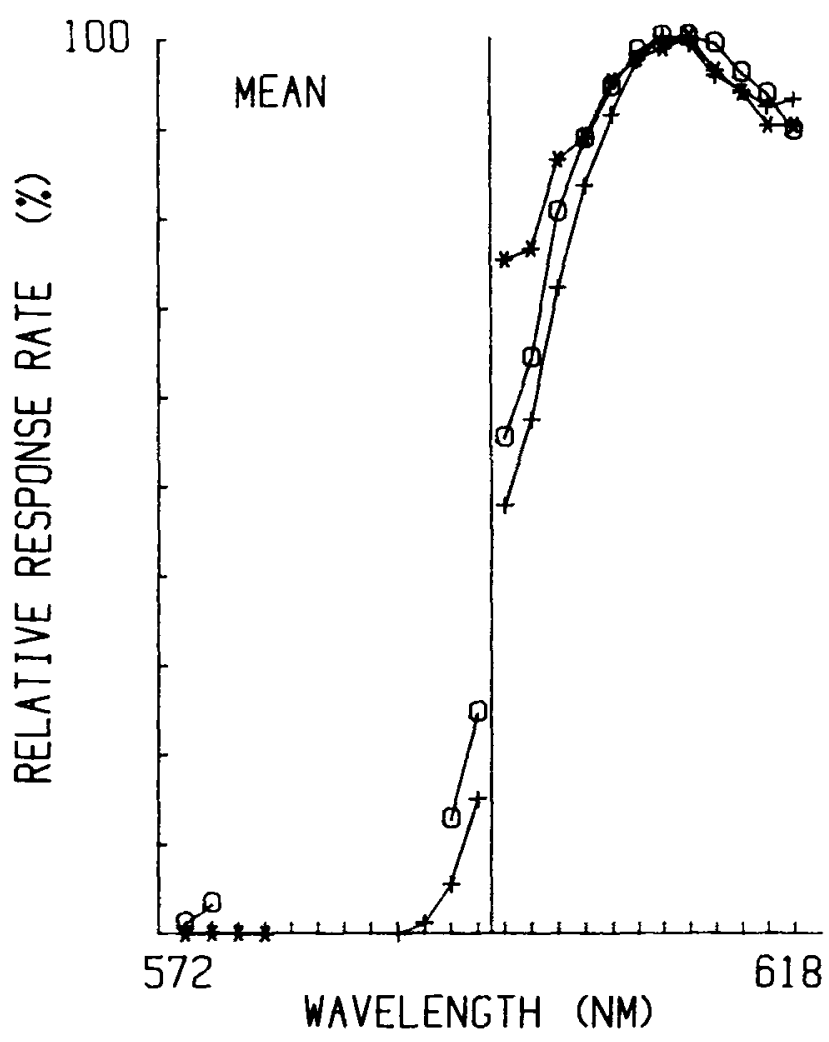

Figure 2. Mean maintained generalization gradients for the 4 birds in Experiment 1. The vertical axis of the gradients represents the mean relative response rate for the first seven consecutive sessions of stable performance during each discrimination condition. Symbols for each condition are an upright cross for Condition 1 , an open circle for Condition 2, and an asterisk for Condition 3. The horizontal axis is scaled in 2-nm divisions. The perpendicular line between values 594 and $596 \mathrm{~nm}$ shows the division between $\mathrm{S}-$ and $\mathrm{S}+$.

removing $S-$ stimuli that were on the border with $S+$ led to increases in $\mathrm{S}+$ response rate of as much as $50 \%$ on average, while leaving other aspects of gradient form unaltered.

\section{Discussion}

With only minor exceptions, the results from Experiment 1 are uniform for all subjects. Discrimination training produced positive dimensional contrast, except in one condition for 1 subject. Although varying somewhat in magnitude for different subjects, the general form of the effects was similar for all birds.

Variation in the spacing of a constant number of $S-$ stimuli did not have a noticeable effect on the form or magnitude of dimensional contrast. The spacing of $\mathrm{S}-$ values, however, did have an effect on responding to some $\mathrm{S}+$ stimuli. Relative response rates increased during $\mathrm{S}+$ stimuli on the border with $S$ - values, as other $S-$ stimuli were moved farther away on the continuum. Thus, changes in the spacing of S- stimuli in Experiment 1 affected discrimination between similar $\mathrm{S}-$ and $\mathrm{S}+$ stimuli, without having a noticeable impact on the peak of $\mathrm{S}+$ responding or on the magnitude of dimensional contrast.

Aspects of the results from Experiment 1 are congruent with current theories of dimensional stimulus control. For example, the decrease in responding to border $S+$ stimuli when the S- set was closely spaced is predicted by the interacting gradient and signal detection models, as well as by D. S. Blough's incremental model. However, the general result of positive dimensional contrast is expected only in the incremental model (see D. S. Blough, 1983).

Other results from Experiment 1 suggest limitations of the incremental model. For instance, the model implies a direct relation between measures of $S-/ S+$ discrimination and the occurrence of dimensional contrast. This prediction derives from the assumption that shared elements between $\mathrm{S}-$ and $\mathrm{S}+$ are responsible for dimensional contrast. It is undoubtedly true that some level of discrimination difficulty between $S-$ and $S+$ is necessary for the production of dimensional contrast (e.g., P. M. Blough, 1980; Hinson, 1988), or at least its measurement. However, the present results indicate that large changes in discrimination difficulty between $\mathrm{S}+$ and $\mathrm{S}-$ do not necessarily affect dimensional contrast as the incremental model seems to suggest.

\section{EXPERIMENT 2}

Experiment 1 showed little change in the form and magnitude of dimensional contrast with variation in the $\mathbf{S}-$ stimulus set. Experiment 2 examined changes in discrimination performance with variation in the set of $S+$ stimuli. In all discrimination conditions, the number and spacing of $S$ - stimuli were held constant, while the number and spacing of $\mathbf{S}+$ stimuli varied across conditions. Experiment 2 should indicate whether positive dimensional contrast is as insensitive to changes in the $\mathrm{S}+$ stimulus set as it was to changes in the S- set.

\section{Method}

Subjects. Three male homing pigeons served as subjects. All 3 had varied training histories that did not include wavelength discrimination training. The subjects were maintained at $80 \%$ of their laboratory, free-feeding weights.

Apparatus. Experiments were carried out in the apparatus used in Experiment 1.

Procedure. The subjects were exposed to a maintained generalization procedure similar to that described in Experiment 1. Stimuli ranged from 572 to $616 \mathrm{~nm}$. Stimuli lower than $596 \mathrm{~nm}$ were designated $S-$, and responses during these stimuli were extinguished. Stimuli higher than or equal to $596 \mathrm{~nm}$ were designated $\mathrm{S}+$, and responses during these values were reinforced according to a variable-interval 60 -sec schedule. For baseline, only $S+$ values appeared throughout the session, whereas for the discrimination conditions, both $\mathrm{S}+$ and $\mathrm{S}-$ values appeared. A single session comprised 12030 -sec stimulus presentations. The sequence of stimuli was determined by a pseudorandom process and changed daily.

Probability of presentation of $S+$ and $S-$ was the same in the discrimination conditions. The number and spacing of $S-$ values were constant across discrimination conditions, whereas the number and spacing of $\mathbf{S}+$ values varied across conditions. Discrimination conditions always included the three $S+$ values adjacent to 
Table 3

Wavelength Values (in $\mathrm{nm}$ ) for $\mathrm{S}+$ and $\mathrm{S}-$, in Each Condition of Experiment 2

\begin{tabular}{ccccc}
\hline & \multicolumn{4}{c}{ S+ Condition } \\
\cline { 2 - 5 }$S-$ & 1 & 2 & 3 & 4 \\
\hline 594 & 596 & 596 & 596 & 596 \\
586 & 598 & 598 & 598 & 598 \\
580 & 600 & 600 & 600 & 600 \\
572 & 604 & & 604 & 612 \\
& 606 & & 606 & 614 \\
& 608 & & 608 & 616 \\
& 612 & & & \\
& 614 & & & \\
\hline
\end{tabular}

the border with S- (i.e., 596, 598, and $600 \mathrm{~nm}$ ). In different conditions, additional $S+$ stimuli, varying in proximity along the continuum from $S-$, were added to the discrimination set. The stimulus values, in nanometers, for $\mathbf{S}+$ and $S-$ during the conditions of Experiment 2 are presented in Table 3.

Each subject was first exposed to the baseline condition. Following this, the subjects received training on the four discrimination conditions. The sequence of conditions for each subject was chosen randomly, without replacement, from a set of predetermined orders. The order of conditions and number of daily sessions in each condition appear in Table 4.

\section{Results}

Figure 3 provides maintained generalization gradients for individual subjects in each discrimination condition of Experiment 2. Each discrimination condition is represented by a function with a unique symbol: an asterisk for Condition 1, a rotated cross for Condition 2, a circle for Condition 3, and an upright cross for Condition 4 . Because there were no obvious differences among subjects in the rate of discrimination learning, all gradients in Figure 3 are taken from the last seven sessions of each discrimination condition. These sessions met the same stability criteria applied in Experiment 1. As in Figures 1 and 2, the gradients in Figure 3 are plotted in relative response rate to minimize the influence of fluctuation in absolute rate. The horizontal axis of each graph is scaled in 2-nm spacings to facilitate comparison with the earlier figures.

The plots for Condition 1 in Figure 3 show that positive dimensional contrast appeared for all subjects. These gradients are characterized by peaks in responding during $\mathrm{S}+$ at $608 \mathrm{~nm}$. Moreover, the difference in response rate between peak and extreme values of $S+$ varies from $10 \%$ and $18 \%$ of maximum responding. This result is very similar to the outcome of Experiment 1.

Figure 3 shows significant changes in the form of the discrimination gradient as a function of discrimination condition. Comparisons can be made for the relative position of border, extreme, and peak values of $\mathrm{S}+$. Responding during the three border stimuli is very similar in Conditions 2, 3, and 4, with the exception of Condition 4 for Bird 5 . In Condition 4, responding during extreme $\mathrm{S}+$ stimuli is about the same as responding during the nearest $\mathrm{S}+$ border stimulus for Birds 5 and 7 , whereas responding is much lower during extreme values for Bird 6. The nonmonotonic gradient form characteristic of positive dimensional contrast appears in both Conditions 1 and 3 for all subjects. Furthermore, for all subjects the peaks of the functions for Conditions 1 and 3 are in different, but consistent, locations along the continuum (viz. $600 \mathrm{~nm}$ for Condition 3 and $608 \mathrm{~nm}$ for Condition 1).

Figure 4 provides a set of mean maintained gradients for the group of subjects in all discrimination conditions of Experiment 2. This figure shows only modest changes in the rate of responding to $S$ - stimuli across conditions, although response rate is lowest in Condition 1. Of more interest is a comparison of relative response rates during $\mathrm{S}+$ stimuli with different spacings. The magnitude of positive dimensional contrast, indicated by the difference in response rate between peak and extreme $\mathbf{S}+$ values, is approximately equal in Conditions 1 and 3 . However, the relative difference in response rate during the peak and border values of $S+$ is much greater in Condition 1 than in Condition 3. Comparison of the sets of border and extreme $\mathrm{S}+$ stimuli also indicates that response rates during extreme values of $S+$ are relatively higher than response rates during border values of $\mathrm{S}+$ in Condition 1 . Finally, the degree of discrimination among the border $\mathrm{S}+$ values differs across conditions. In Condition 1 , with $S+$ stimuli spread across the entire range, the separation between adjacent values of $S_{-}$and $S+$ is smaller.

\section{Discussion}

Experiment 2 shows that dimensional stimulus control gradients change markedly with variations in the spacing of the $\mathbf{S}+$ stimulus set. This result contrasts with the relative insensitivity of gradient form to variations in the $S-$ stimulus set as shown in Experiment 1. In Experiment 2, the discrimination profile changed as the range of $\mathrm{S}+$ stimuli increased. For example, peak responding within the $\mathrm{S}+$ set moved away from the $\mathrm{S}-$ border as the range was extended from Condition 3 to Condition 1 . But the spacing of $\mathbf{S}+$ values within a given range also made a difference. For instance, consider the three $\mathrm{S}+$ stimuli that were presented near the $S-$ border in each condition. Discrimination of these from $S-$ was relatively lower in Condition 1 than in Condition 4, even though the range

Table 4

Order of Conditions and Number of Sessions for Each Condition of Experiment 2

\begin{tabular}{cl}
\hline Bird & Condition (Sessions) \\
\hline 5 & Baseline (1-21), C3 $(22-49)$, C1 (50-77), C2 (78-105), C4 (106-133) \\
6 & Baseline (1-21), C4 (22-49), C3 (50-77), C2 (78-105), C1 (106-133) \\
7 & Baseline (1-21), C2 (22-49), C4 (50-77), C1 (78-105), C3 (106-133) \\
\hline
\end{tabular}




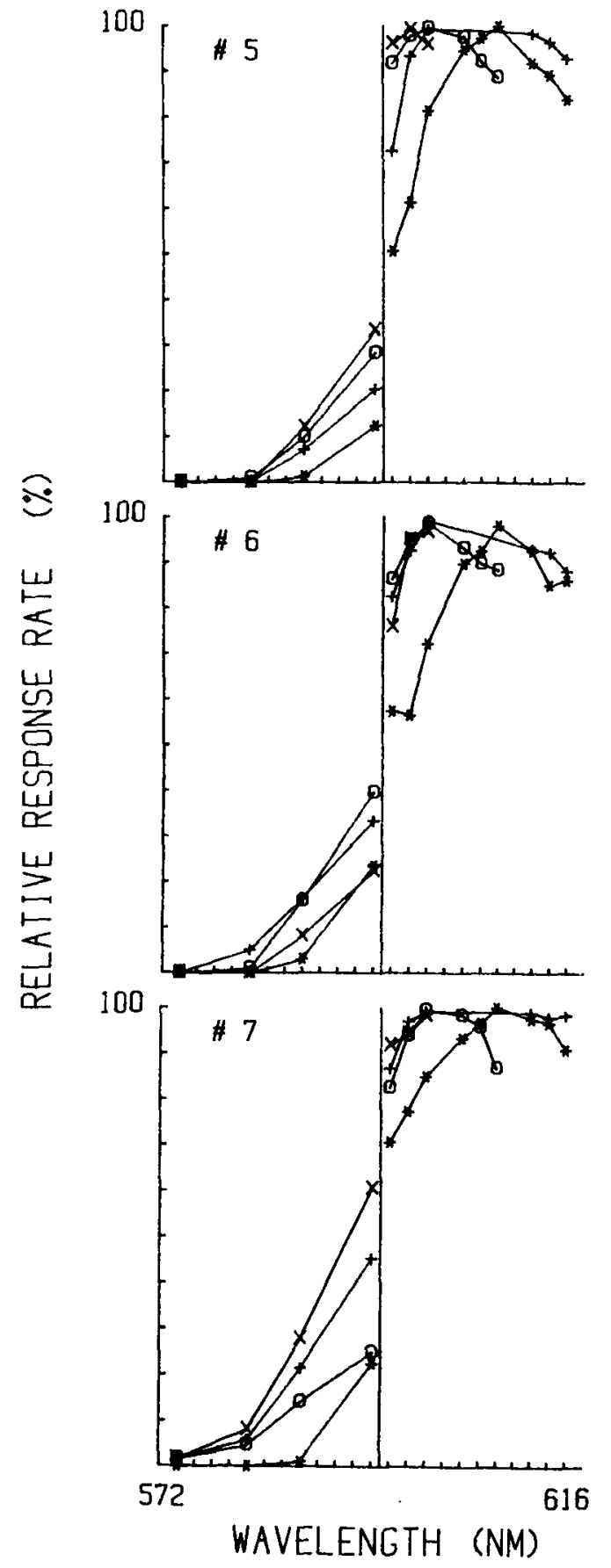

Figure 3. Maintained generalization gradients for the 3 birds in Experiment 2. The vertical axis of the gradients represents the mean relative response rate for the last seven sessions of each discrimination condition. Symbols for each condition are an asterisk for Condition 1, a rotated cross for Condition 2, an open circle for Condition 3, and an upright cross for Condition 4. The horizontal axis is scaled in 2-nm divisions. The perpendicular line between values 594 and $596 \mathrm{~nm}$ shows the division between $\mathrm{S}-$ and $\mathrm{S}+$. of $\mathrm{S}+$ stimuli in the two conditions was identical. Thus the intermediate $\mathrm{S}+$ values in Condition 1 had an influence apart from variations in range.

\section{GENERAL DISCUSSION}

D. S. Blough (1983) described various accounts of dimensional stimulus control and their applicability to dimensional contrast effects. The interacting gradient model originally proposed by Spence (1937) has difficulty explaining dimensional contrast. Similarly, in its present form, the signal detection model (e.g., Boneau \& Cole, 1967) does not predict dimensional contrast effects. Perhaps future modifications of these models will be more satisfactory, but this remains speculative.

Two more acceptable candidate models are also described by D. S. Blough (1983). One is the incremental model proposed by D. S. Blough (1975). The incremental model successfully accounts for a wide range of reported data (e.g., D. S. Blough, 1975; P. M. Blough, 1980; Essock \& D. S. Blough, 1977). Moreover, simulation studies indicate that the model works admirably well (e.g., D. S. Blough, 1975; Rowe, 1981). A second candidate is an opponent process model, which is fundamentally equivalent to the neural unit approach suggested by several authors (e.g., Catania \& Gill, 1964; Hinson \& Malone, 1980; Malone \& Staddon, 1973; Rowe, 1981). The opponent process model has not been formally developed, but is based on reciprocal excitatory/inhibitory interactions, such as those occurring in the receptive fields of sensory systems (e.g., Ratliff, 1965).

Although not designed as a test of any specific theory, the current studies do address some general predictions of the incremental model. For instance, the model predicts direct variation between dimensional contrast and similarity of S- and S+ (D. S. Blough, 1975; P. M. Blough, 1980). However, Experiment 1 showed that the form and magnitude of dimensional contrast remained constant despite large changes in similarity of S- and S + . In addition, the incremental model predicts that dimensional contrast should be largely independent of the number and spacing of stimuli (Essock \& D. S. Blough, 1977). Nonetheless, Experiment 2 showed that dimensional contrast was dependent on both these variables.

Taken together, the implication of these experiments is that range and spacing of values within the S+ stimulus set may be as important as similarity between $S-$ and $\mathrm{S}+$ in determining dimensional contrast. Although dimensional contrast depends on the distance between $S-$ and $\mathrm{S}+$, absolute distance along the physical continuum is not the critical variable. Rather, relative distance, as defined by the range and spacing of stimuli, appears more important (see also Hinson \& Lockhead, 1986). Therefore, models of dimensional contrast should probably be based on relative, rather than absolute, measures of similarity. 


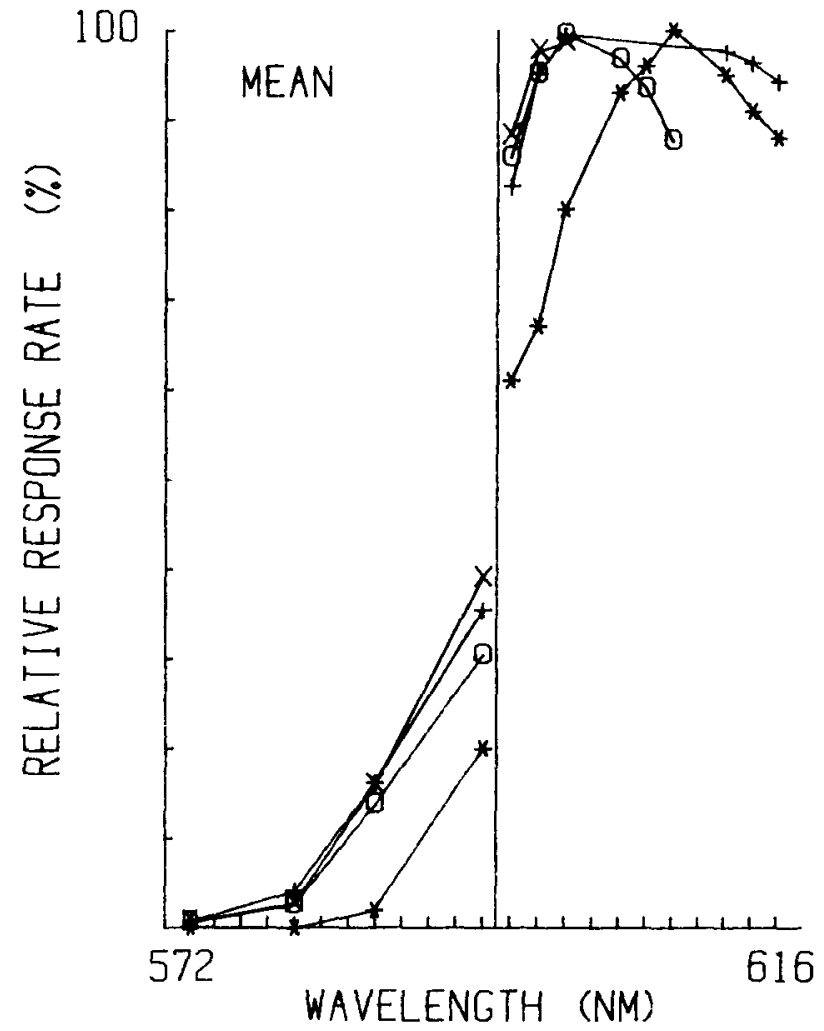

Figure 4. Mean maintained generalization gradients for the group of 3 birds in Experiment 2. The vertical axis of the gradients represents the mean relative response rate for the last seven sessions of each discrimination condition. Symbols for each condition are an asterisk for Condition 1, a rotated cross for Condition 2, an open circle for Condition 3 , and an upright cross for Condition 4. The horizontal axis is scaled in 2 -nm divisions. The perpendicular line between values 594 and $596 \mathrm{~nm}$ shows the division between $S-$ and S+.

The outcomes of the present experiments can be compared with similar effects that appear to reflect relative, rather than absolute, stimulus ordering. One example is the central tendency effect reported in human stimulus generalization (e.g., Thomas \& Jones, 1962; Thomas, Strub, \& Dickson, 1974). In these studies, the peak of generalization gradients obtained during extinction tests depends on the distribution of test stimuli, rather than the difference between training and test stimuli along the physical dimension. Stimuli appear to be judged according to a relational category organization consistent with adaptation level theory (see also Thomas, Windell, Williams, \& White, 1985).

It is tempting to entertain the notion that dimensional contrast effects reflect some sort of relational categorization of stimuli within the range of values presented. Accordingly, peak response rates might occur during the most typical or average $S+$ value, perhaps determined by both the range and frequency of presentation of all $\mathrm{S}+$ values. It follows that the peak stimulus value would not be the most distinct or discriminable from $\mathrm{S}-$, but one that is intermediate within the range of variation of the S+ set.

Despite its appeal, one should be wary before extending the central tendency interpretation to the present results. Beyond differences in subjects and procedures, several aspects of studies confined to the transient generalization testing technique suggest caution. First, the adaptation level account used to explain the central tendency effect does not appear to be a general explanation of discrimination training effects, even in the human paradigm (e.g., Newlin, Rodgers, \& Thomas, 1979). Second, such an account does not readily explain bimodal postdiscrimination gradients obtained with multiple stimulus training in either human studies (e.g., White \& Thomas, 1979) or animal studies (e.g., Kalish \& Guttman, 1959). Third, pigeons do not seem as sensitive as humans to test stimulus variation during the extinction phase of the transient generalization test (e.g., Stevenson, 1966).

Nonetheless, a virtue of the central tendency explanation is that it is easy to examine experimentally. For example, an adequate test would be provided by a discrimination involving a set of $S+$ stimuli bounded on both ends of the continuum by $\mathrm{S}-$. Two distinguishable outcomes are likely in this setting. If the stimulus continuum is psychologically uniform, then a single peak in $S+$ responding between the $S-$ boundaries would be an outcome consistent with the central tendency account. However, if peaks in $\mathbf{S}+$ responding are observed at both $\mathrm{S}$ - borders, then the prior explanation would be ruled out. Either outcome would clarify the interpretation of stimulus range and spacing variables.

\section{REFERENCES}

BLough, D. S. (1975). Steady-state generalization and a quantitative model of operant generalization and discrimination. Jourmal of Experimental Psychology: Animal Behavior Processes, 104, 3-21.

BLough, D. S. (1983). Alternative accounts of dimensional stimulus control. In M. Commons, R. Herrnstein, \& A. Wagner (Eds.), Quantitative analyses of behavior (Vol. 4, pp. 59-72). Cambridge, MA: Ballinger.

BLough, P. M. (1980). Behavioral and dimensional contrast in rats. Journal of the Experimental Analysis of Behavior, 33, 345-357.

BLough, P. M., \& Blough, D. S. (1985). Sequential effects in dimensional contrast. Joumal of the Experimental Analysis of Behavior, 44, 233-244.

Boneau, C. A., Cole, J. L. (1967). Decision theory, the pigeon, and the psychophysical function. Psychological Review, 74, 123-135.

Catania, A. C., \& Gill, C. A. (1964). Inhibition and behavioral contrast. Psychonomic Science, 1, 257-258.

Essock, S. M., \& BLough, D. S. (1977). Effects of stimulus spacing on steady-state gradients of inhibitory stimulus control. Animal Leaming \& Behavior, 5, 174-176.

FARTHING, G. W. (1974). Behavioral contrast with multiple positive and negative stimuli on a continuum. Joumal of the Experimental Analysis of Behavior, 22, 419-425.

HeinemanN, E., \& Chase, S. (1975). Stimulus generalization. In W. K. Estes (Ed.), Handbook of learning and cognitive processes (Vol. 2, pp. 305-349). Hillsdale, NJ: Erlbaum.

Hinson, J. M. (1988). Absolute and relative measures of dimensional contrast. Journal of the Experimental Analysis of Behavior, 50 , 249-260.

Hinson, J. M., LockheAd, G. R. (1986). Range effects in succes- 
sive discrimination. Journal of Experimental Psychology: Animal Behavior Processes, 12, 270-276.

Hinson, J. M., \& MALONE, J. C., JR. (1980). Local contrast and maintained generalization. Journal of the Experimental Analysis of Behavior, 34, 263-272.

Honig, W. K., \& Urcuioli, P. J. (1981). The legacy of Guttman and Kalish (1956): 25 years of research on stimulus generalization. Journal of the Experimental Analysis of Behavior, 36, 405-445.

Kalish, H. I., \& GuTtMan, N. (1959). Stimulus generalization after training on three stimuli: A test of the summation hypothesis. Journal of Experimental Psychology, 57, 268-272.

Malone, J. C., JR. (1975). Stimulus-specific contrast effects during operant discrimination learning. Journal of the Experimental Analysis of Behavior, 24, 281-289.

MALONE, J. C., JR., \& RoWE, D. W. (1981). Local contrast, local dimensional effects, and dimensional contrast. In M. Commons \& J. A. Nevin (Eds.), Quantitative analyses of behavior (Vol. 1, pp. 365-390). Cambridge, MA: Ballinger.

Malone, J. C., JR., Staddon, J. E. R. (1973). Contrast effects in maintained generalization gradients. Journal of the Experimental Analysis of Behavior, 19, 167-179.

Newlin, R. J., Rodgers, J. P., \& Thomas, D. R. (1979). Two determinants of the peak shift in human voluntary stimulus generalization. Perception \& Psychophysics, 25, 478-486.

RATLifF, F. (1965). Mach bands: Quantitative studies on neural networks in the retina. San Francisco: Holden-Day.

REYNOLDS, G. S. (1961). Contrast, generalization, and the process of discrimination. Journal of the Experimental Analysis of Behavior, 4, 289-294.
RowE, D. W. (1981). Multiple schedule interactions and a model of operant discrimination. Unpublished doctoral dissertation, University of Tennessee, Knoxville.

Rowe, D. W., \& MALONE, J. C., JR. (1981). Multiple schedule interactions and discrimination. Animal Learning \& Behavior, 9, 115-126

SPENCE, K. W. (1937). The differential response in animals to stimul varying within a single dimension. Psychological Review, 44, 430-444.

Stevenson, J. G. (1966). Stimulus generalization: The ordering and spacing of test stimuli. Journal of the Experimental Analysis of Behavior, 9, 457-468.

Thomas, D. R., \& JoNes, C. G. (1962). Stimulus generalization as a function of the frame of reference. Joumal of Experimental Psychology, 64, 77-80.

Thomas, D. R., Strub, H., \& Dickson, J. F. (1974). Adaptation level theory and the central tendency effect in stimulus generalization. Journal of Experimental Psychology, 103, 446-474.

Thomas, D. R., Windell, B. T., Williams, J. L., \& White, K. G. (1985). Stimulus presentation frequency in brightness discrimination and generalization: A test of adaptation level and signal detection interpretations. Perception \& Psychophysics, 37, 243-248.

White, K. G., \& Thomas, D. R. (1979). Postdiscrimination stimulus generalization in humans: An extension of Galizio and Baron. Perception \& Psychophysics, 7, 564-565.

(Manuscript received April 27, 1988; revision accepted for publication July 25,1988 .) 